\title{
Interscalene Block for Analgesia in Orthopedic Treatment of Shoulder Trauma: Single-Dose Liposomal Bupivacaine versus Perineural Catheter
}

\section{Andrzej P Kwater (D) \\ Nadia Hernandez \\ Carlos Artime \\ Johanna Blair de Haan}

Department of Anesthesiology, McGovern Medical School at UTHealth, Houston, TX, USA
Correspondence: Andrzej P Kwater Department of Anesthesiology and Perioperative Medicine, The University of Texas MD Anderson Cancer Center, 1515 Holcombe Blvd., Unit 409, Houston, TX, 77030, USA

Tel +0I 7/3 7926911

Email apkwater@mdanderson.org
Background: Interscalene brachial plexus block is frequently utilized to provide perioperative analgesia to patients undergoing shoulder surgery to optimize recovery, minimize opioid consumption, and decrease overall hospital length of stay. The use of an indwelling perineural interscalene catheter provides extended analgesia and is efficacious in managing severe postoperative pain following major shoulder surgery. Currently, the only alternative to perineural catheters for extended analgesia with interscalene block involves the perineural infiltration of liposomal bupivacaine. However, there is limited published data regarding the overall analgesic effectiveness of using interscalene liposomal bupivacaine in the setting of shoulder surgery.

Methods: We performed a retrospective review of 43 patients in the acute trauma setting who underwent major shoulder surgery and received extended analgesia via perioperative interscalene brachial plexus block with either an indwelling continuous catheter or single-dose liposomal bupivacaine to determine if comparable analgesia can be achieved. The primary outcomes of interest were postoperative pain scores and opioid consumption. Due to the ability to titrate and bolus local anesthetic infusions to a desired clinical effect, we hypothesized that opioid consumption and pain scores would be lower when using the continuous catheter technique.

Results: After statistical analysis, our results demonstrated no significant difference between the two techniques in regards to opioid consumption as well as numeric pain scores during the 48hour postoperative period, but did note a higher rate of complications with patients who received perineural interscalene continuous catheters. Secondary outcomes showed an increase in time required to complete the regional block procedure with the use of indwelling catheters.

Conclusion: Interscalene brachial plexus block with liposomal bupivacaine may be a viable alternative to indwelling continuous catheters for providing extended analgesia in patients undergoing major shoulder surgery.

Keywords: brachial plexus, analgesia, perineural, postoperative pain, retrospective

\section{Introduction}

Major shoulder surgery, including total shoulder arthroplasty and rotator cuff repair, is characterized by severe acute postoperative pain necessitating a multidisciplinary approach to achieve effective multimodal pain management. Inadequate postoperative analgesia can lead to prolonged hospital stays, delayed recovery, increased costs, as well as persistent pain in the acute setting, which places patients at risk of developing chronic pain as well as adhesive capsulitis, also known as "frozen shoulder". Regional anesthesia has had a significant impact on perioperative outcomes in orthopedic surgery including improved postoperative analgesia and rehabilitation, reduced opioid requirements, shortened hospital length of stay, and better functional outcomes and range of motion. ${ }^{1,2}$ 
In the setting of shoulder surgery, interscalene brachial plexus block (ISB) has been utilized as an effective analgesic technique in the management of postoperative pain. ${ }^{3,4}$ Specifically, ISB with a continuous catheter system (ISB-CC) has been shown to be an effective analgesic technique in the postoperative setting following major shoulder surgery by improving duration of analgesia and overall patient satisfaction, reducing hospital length of stay, and minimizing opioid consumption., ${ }^{5,6}$ Despite these proven benefits, however, the literature suggests that peripheral nerve blocks are overall under-utilized in both inpatient and ambulatory orthopedic surgeries. ${ }^{1}$ This could be due in part to the time required to perform peripheral nerve blocks as well as the increased labor associated with managing perioperative acute pain, particularly with indwelling catheters. Single-shot peripheral nerve blocks, although technically easier and less timeconsuming, are limited by the duration of analgesia, which typically subsides within the initial 24 hours following surgery, even with the use of additives. ${ }^{7,8}$ This ultimately leads to the potential for significant rebound pain and prolonged hospital stays due to inadequate pain control in the perioperative period. ${ }^{9}$

As a potential alternative for extended analgesia in the form of a single-shot peripheral nerve block, in 2018, the US Food and Drug Administration (FDA) approved liposomal bupivacaine (Exparel: Pacira BioSceinces Inc., Parsippany, NJ, USA) for use in ISB with a theoretical analgesic effectiveness of 72 hours following initial injection. Liposomal bupivacaine is an injectable suspension that provides a slow, continuous release of bupivacaine over an extended period of time following administration. As a result, the use of liposomal bupivacaine for ISB has garnered significant interest as a potential alternative to the use of indwelling catheters for extended analgesia.

In the setting of shoulder surgery, there is limited published data comparing the analgesic effectiveness of ISB-CC and interscalene liposomal bupivacaine (ISB-LB). Since there is no clear consensus on which method could be more effective for extended analgesia in the perioperative period, the decision to use a particular technique often falls on availability of resources, clinician preference, and overall workflow. This study examined the postoperative course of patients in the trauma setting who received ISB following major shoulder surgery. Our primary objective was to compare the efficacy of ISB-CC and ISB-LB by evaluating numeric pain scores and opioid consumption during the postoperative period. Due to the ability to titrate and bolus local anesthetic infusions to a desired clinical effect, we hypothesized that opioid consumption and postoperative pain scores during the initial 48-hour postoperative period would be lower for ISB-CC as compared to ISB-LB in shoulder trauma patients.

\section{Materials and Methods}

This is a retrospective case-control study that examined postoperative outcomes following ISB with either an ISBCC or ISB-LB in the setting of open shoulder surgery. The study was conducted at Memorial Hermann Hospital Texas Medical Center (Houston, TX, USA), which serves as one of the busiest Level 1 trauma centers in the United States. University of Texas Health Science Center Institutional Review Board (IRB) exemption status (HSCMS-19-0186) and hospital approval by the Memorial Hermann Health System (Clinical Innovation and Research Institute) was granted to begin the research study. IRB exemption status was granted since no risks were associated with the retrospective nature of the study and data was gathered through electronic medical record review following a patient's treatment course, with no effect on clinical care or outcomes. Thus, patient consent to review medical records was not required and all collected data was maintained and stored securely without any identifiers to maintain patient data confidentiality. In addition, this study was performed in compliance with the principles of the Declaration of Helsinki.

Initially, we identified all patients receiving ISB in the setting of open shoulder surgery during a 14-month period that extended from January 2018 until February 2019. Based on electronic medical record review, each patient was screened for specific inclusion and exclusion criteria for enrollment in the study. Inclusion criteria were patients 18 years or older, American Society of Anesthesiologists (ASA) Physical Status $1-4$, undergoing open shoulder surgery, and receiving either perioperative ISB-CC or ISB-LB. Exclusion criteria included the following: 1) contraindications to regional anesthesia or patient refusal, 2) history of hypersensitivity reaction to local anesthetic, 3) timing of nerve blockade that did not correspond with the day of surgery, 4) patients undergoing additional surgical interventions or regional anesthetic procedures within a 48-hour period, and 5) patients with a history of chronic pain with documented opioid prescriptions (eg, hydrocodone, oxycodone, morphine, hydromorphone, methadone) in our Texas Prescription Monitoring Program (texas.pmpaware.net) records prior to admission to the hospital. 


\section{Peripheral Nerve Blockade}

All nerve blocks were performed with ASA standard monitoring under ultrasound-guidance as well as supervision by a regional and acute pain fellowship-trained anesthesiologist. Each patient was a candidate for regional anesthesia with no contraindications to placement of peripheral nerve block.

\section{Interscalene Indwelling Catheter Technique}

For placement of the ISB-CC, a high-frequency linear transducer ultrasound probe was placed in the supraclavicular fossa in the transverse plane to identify the subclavian artery and brachial plexus. The brachial plexus was traced cranially until the $\mathrm{C} 5$ and $\mathrm{C} 6$ nerve roots were identified in short-axis between the anterior and middle scalene muscles. ${ }^{10}$ The dorsal scapular and long thoracic nerves were identified in the muscle belly of the middle scalene to avoid trauma to these structures. An 18-gauge Tuohy needle was advanced in-plane using hydrodissection with normal saline until the needle was lateral to the brachial plexus sheath (Figure 1). $20 \mathrm{~mL}$ of $0.25 \%$ bupivacaine hydrochloride was then injected in $5 \mathrm{~mL}$ aliquots while confirming negative aspiration prior to each injection. Subsequently, a 20-gauge perineural catheter was advanced through the needle, and catheter tip placement confirmed by ultrasound visualization of injectate spread around the nerve roots through the catheter. The catheter was then secured with topical skin adhesive (Dermabond) at the catheter insertion site to prevent leaking, liquid adhesive (Mastisol), and steri-strips followed by a chlorhexidine gluconate securement dressing (3M CHG) to prevent catheter dislodgement or migration.

Following catheter placement, a continuous infusion of $0.2 \%$ ropivacaine (basal rate of $6-8 \mathrm{~mL} / \mathrm{hr}$ ) was initiated with bolus-dosing settings (bolus dose $3 \mathrm{~mL}$, lockout time 30 minutes, and 1-hour limit set to baseline hourly infusion volume plus $6 \mathrm{~mL}$ ). The use of $0.2 \%$ ropivacaine for the continuous infusion was based on its efficacy in providing postoperative analgesia in comparison to other local anesthetics. ${ }^{11}$ The catheter infusion was continued for 24 to 96 hours based on clinical outcome or hospital discharge. The infusion pump was adjusted by the acute pain service by increasing or decreasing the rate, discontinuing the infusion and/or changing the bolus dose as indicated. If deemed appropriate, patients were given the option to be discharged with an elastomeric pump, which provided a continuous infusion of $0.2 \%$ ropivacaine at $6 \mathrm{~mL} / \mathrm{hr}$ until completion of the allotted volume (generic allocated volume of $400 \mathrm{~mL} 0.2 \%$ ropivacaine provided approximately 66 hours of continuous infusion). Followup after discharge was maintained via telephone to ensure uneventful removal of the catheter, resolution of nerve blockade without complications, and a smooth transition to oral analgesics.

\section{Interscalene Single-Shot Block with Liposomal Bupivacaine Technique}

For single-shot ISB-LB, the same technique was used for identifying the brachial plexus at the level of the $\mathrm{C} 5$ and

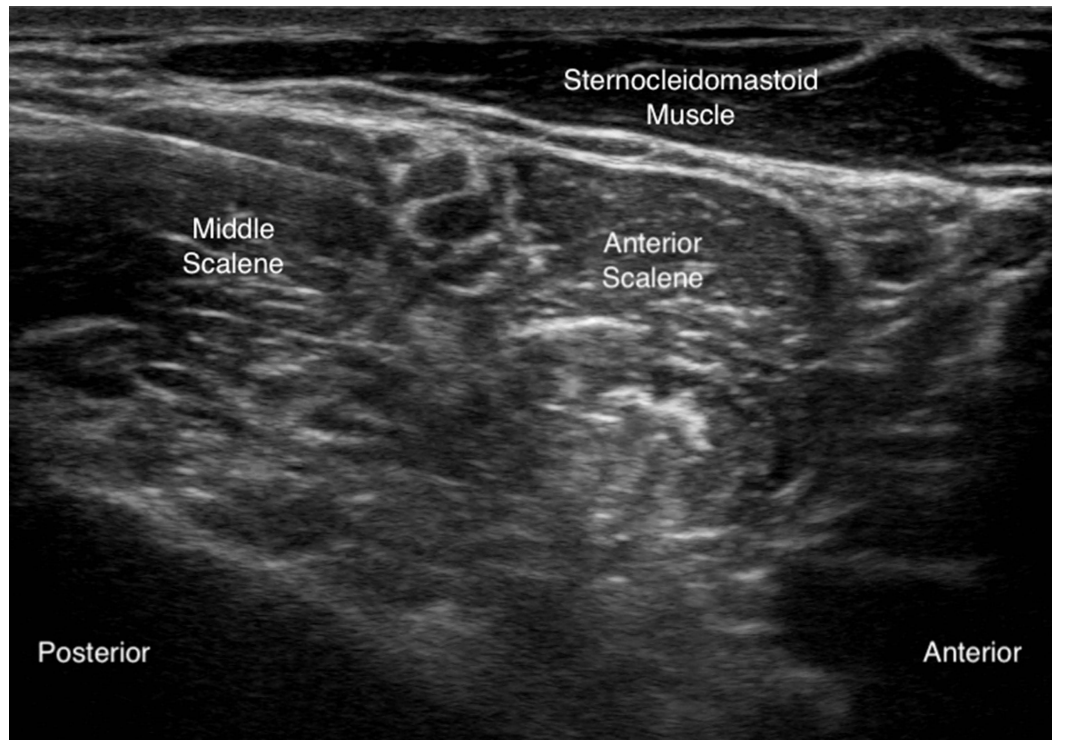

Figure I Ultrasound view of interscalene nerve block depicting orientation of brachial plexus in relation to surrounding structures (sternocleidomastoid muscle, middle scalene muscle, anterior scalene muscle). 
C6 nerve roots. A 20-gauge blunt-tip echogenic needle was advanced in-plane using hydrodissection with normal saline until the needle was positioned lateral to the brachial plexus sheath. After negative aspiration, a combined solution of $10 \mathrm{~mL}$ of $1.3 \%$ liposomal bupivacaine and $10 \mathrm{~mL}$ of $0.25 \%$ bupivacaine hydrochloride was injected slowly through the needle in $5 \mathrm{~mL}$ aliquots.

\section{Block Assessment}

Blocks were performed after a neurological exam was completed and the patient was cleared by the orthopedic trauma surgeon. Postoperative pain was assessed by nursing staff using a verbal numeric pain scale (NPS, 0-10 range). Block success was ascertained by a combination of notable decrease in shoulder pain to mild (0-3 on NPS) as well as motor examination after the intervention.

During the postoperative period, each patient was evaluated by the acute pain service twice a day and received a standardized multimodal pain regimen of oral medications during the inpatient stay. With consideration of each patient's comorbidities, the multimodal pain regimen consisted of acetaminophen $1000 \mathrm{mg}$ every 6 hours, celecoxib $200 \mathrm{mg}$ every 12 hours, gabapentin $300 \mathrm{mg}$ every 8 hours, tramadol $50 \mathrm{mg}$ every 6 hours, methocarbamol $1000 \mathrm{mg}$ every 8 hours, and oxycodone $5 \mathrm{mg}$ or $10 \mathrm{mg}$ every 4 hours as needed for moderate (4-6 on NPS) or severe pain (7-10 on NPS), respectively.

\section{Data Collection}

For each patient included in the study, a review of the electronic medical record was performed to collect data that included type of intervention, pain scores, and opioid consumption during the 48 -hour postoperative period. The 48-hour postoperative period was set as the timeline for data analysis because many patients were discharged on postoperative day 2 (POD 2), resulting in inconsistent and unreliable documentation of clinical outcomes for comparison after that designated time period. In addition, demographic data (age, gender, BMI, ASA classification) as well as type of shoulder surgery were recorded for each patient (Tables 1 and 2). Duration of time required to complete the regional nerve block technique was also noted. Any perioperative events or complications related to each intervention were identified.

Following ISB, pain scores were reported on a NPS (010 , with 0 being no pain and 10 being the worst pain imaginable) and collected at approximate postoperative time points ( 6 hours, 12 hours, 24 hours, 36 hours, and 48 hours) within a range of 1-2 hours based on nursing staff reports. For any patients that received ISB during the preoperative period, these time points of pain scores were measured from the patient's arrival to the post-anesthesia

Table I Patient Demographic Data

\begin{tabular}{|c|c|c|c|}
\hline & ISB-CC & ISB-LB & P-value \\
\hline Age, years (Mean $\pm S D$ ) & $54.1 \pm 3.3$ & $52.6 \pm 3.4$ & $0.764^{\mathrm{t}}$ \\
\hline BMI, $\mathrm{kg} / \mathrm{m}^{2}($ Mean $\pm \mathrm{SD})$ & $29.6 \pm 1.5$ & $29.8 \pm 1.6$ & $0.94 I^{t}$ \\
\hline \multicolumn{4}{|l|}{ Gender (Male/Female) } \\
\hline Male (n, \%) & II (57.9\%) & 15 (62.5\%) & $0.76^{\mathrm{c}}$ \\
\hline Female (n, \%) & $8(42.1 \%)$ & $9(37.5 \%)$ & \\
\hline \multicolumn{4}{|l|}{ ASA Physical Status (Median, IQR)) } \\
\hline I $(n, \%)$ & $0(0 \%)$ & $2(8.3 \%)$ & \\
\hline $2(\mathrm{n}, \%)$ & $6(31.6 \%)$ & $8(33.3 \%)$ & $0.4 I^{\text {wrs }}$ \\
\hline $3(n, \%)$ & $12(63.2 \%)$ & $13(54.2 \%)$ & \\
\hline $4(n, \%)$ & I (5.2\%) & I (4.2\%) & \\
\hline
\end{tabular}

Abbreviations: t, Student's $t$-test; wt, Welch's $t$-test; c, chi-squared test; wrs, Wilcoxon rank sum; IQR, interquartile range; $n$, number of patients; SD, standard deviation.

Table 2 List of Surgical Procedures Among Study Groups

\begin{tabular}{|l|c|c|}
\hline Type of Surgical Intervention & ISB-CC Group, $\mathbf{n}$ (\% of Total) & ISB-LB Group, $\mathbf{n}(\%$ of Total) \\
\hline ORIF Proximal Humerus & $8(42.0)$ & $6(25.0)$ \\
Total Shoulder Arthroplasty & $3(15.8)$ & I (4.2) \\
Reverse Total Shoulder Arthroplasty & $3(15.8)$ & $8(33.2)$ \\
ORIF Greater Tuberosity & $2(10.5)$ & $0(0)$ \\
ORIF Clavicle/Scapula/Acromion & $1(5.3)$ & $0(0)$ \\
Rotator Cuff Repair & I (5.3) & $5(20.8)$ \\
ORIF Glenoid & I (5.3) & I (4.2) \\
ORIF Acromion & $0(0)$ & $1(4.2)$ \\
Shoulder Disarticulation & $0(0)$ & $1(4.2)$ \\
Shoulder Hemi-arthroplasty & $0(0)$ & $1(4.2)$ \\
\hline
\end{tabular}

Abbreviations: ORIF, open reduction internal fixation; n, number of cases. 
care unit (PACU) following surgery. Postoperative opioid consumption was also collected and converted to morphine milligram equivalents (MME) using a set of recommended conversion factors (Table 3). These MME conversion factors are based on published guidelines gathered from a series of international sources and provided a consistent approach to quantifying equivalent opioid consumption. ${ }^{12}$ For postoperative day 0 (POD 0), any preoperative medications (eg, tramadol, oxycodone, hydrocodone, morphine), long-acting intraoperative opioids (eg hydromorphone), and postoperative opioids (eg, tramadol, hydrocodone, oxycodone, codeine, hydromorphone, morphine) were included in the measured total for opioid consumption. The total dose of short-acting opioids (eg, fentanyl) as well as any analgesic adjuvants (eg, ketamine, dexmedetomidine) given intraoperatively or in the PACU were excluded and provided as a separate data analysis (Table 4).

\section{Statistical Analysis and Outcomes}

All eligible patients that met inclusion criteria were included in the data analysis. Primary outcomes measured

Table 3 Opioid Conversion Chart - Morphine Milligram Equivalents (MME)

\begin{tabular}{|l|c|c|}
\hline & Units & $\begin{array}{c}\text { Conversion } \\
\text { Factor }\end{array}$ \\
\hline $\begin{array}{l}\text { Oral Opioid } \\
\text { Codeine }\end{array}$ & $\mathrm{mg}$ & 0.15 \\
Tramadol & $\mathrm{mg}$ & 0.1 \\
Hydrocodone & $\mathrm{mg}$ & $\mathrm{I}$ \\
Oxycodone & $\mathrm{mg}$ & 1.5 \\
Morphine & $\mathrm{mg}$ & $\mathrm{I}$ \\
Hydromorphone & $\mathrm{mg}$ & 4 \\
\hline Intravenous Opioid & & \\
Morphine & $\mathrm{mg}$ & 3 \\
Hydromorphone & $\mathrm{mg}$ & 15 \\
Fentanyl & $\mathrm{mcg}$ & 0.2 \\
\hline
\end{tabular}

Abbreviations: mg, milligrams; mcg, micrograms.

Table 4 Perioperative Medication Administration

\begin{tabular}{|l|c|c|c|}
\hline & ISB-CC & ISB-LB & P-value \\
\hline Fentanyl, mcg (Mean \pm SD) & $250 \pm 24.5$ & $237.5 \pm 17.6$ & $0.68 I^{\mathrm{wt}}$ \\
Ketamine (n, \%) & $10 / 19,53 \%$ & $16 / 24,67 \%$ & $0.35^{\mathrm{c}}$ \\
Dexmedetomidine (n, \%) & $6 / 19,32 \%$ & $1 \mathrm{I} / 24,46 \%$ & $0.34^{\mathrm{c}}$ \\
\hline
\end{tabular}

Abbreviations: wt, Welch's $t$-test; c, chi-squared test; $\mathrm{n}$, number of patients; mcg, microgram; SD, standard deviation. for this study were postoperative pain scores and opioid consumption during the 48-hour period following surgery.

Normally distributed continuous variables (eg, age, BMI, intraoperative fentanyl administration) were compared using Student's $t$-test or Welch's $t$-test. Categorical variables (intraoperative dexmedetomidine or ketamine administration, gender) were compared with Chi-squared tests. Wilcoxon rank-sum was used to evaluate nonnormally distributed data, including postoperative MME totals and interval pain scores, as well as time required to complete the regional nerve block. Level of significance $\mathrm{P}<0.05$ was used during statistical analysis to establish statistically significant differences.

Of note, at the time of planning our retrospective study in 2018, there was no evidence-based measure of effect size in terms of magnitude and variance to perform an a priori sample size calculation. Therefore, the sample size was determined by retrospective convenience sampling. ${ }^{13}$ Ultimately, our sample size was limited to a designated 14month period based on a specific clinical presentation and adherence to inclusion as well as exclusion criteria from a single, level-one trauma center institution.

\section{Results}

\section{Patient Demographic and Perioperative Data}

During the study period, 47 patients met inclusion criteria to be enrolled in our study. Of those 47 patients, 2 patients with chronic pain, 1 patient with surgical intervention within 48 hours of ISB, and 1 patient who received ISB for pain control 2 days prior to the day of surgery were excluded. Therefore, 43 total patient charts were reviewed for data collection, with 19 patients receiving ISB-CC and 24 patients receiving ISB-LB.

Of the total 43 patients, 4 patients (1 from ISB-CC group, 3 from ISB-LB group) received ISB preoperatively on the day of surgery, while all other patients received ISB during the immediate postoperative period in the PACU. Of note, 2 patients from the ISB-LB group received combined interscalene - supraclavicular brachial plexus blocks to provide additional analgesia to the distal humerus. ${ }^{14}$ With respect to the type of shoulder surgery, each patient from the two groups underwent surgical intervention as listed by the subset of major shoulder surgeries in Table 2 .

For the ISB-CC group, there were 2 patients that required an increase in $0.2 \%$ ropivacaine infusion rate from $6 \mathrm{~mL} / \mathrm{hr}$ to $8 \mathrm{~mL} / \mathrm{hr}$ to improve analgesic effect and 
1 patient required the infusion to be decreased from $6 \mathrm{~mL} /$ hr to $4 \mathrm{~mL} / \mathrm{hr}$ to attenuate motor blockade. In addition, a total of 6 patients from the ISB-CC group were given a bolus (10-20 mL of $0.25 \%$ bupivacaine hydrochloride, $0.2 \%$ ropivacaine, or $0.5 \%$ ropivacaine) prior to removal of the indwelling catheter in order to provide extended analgesia. Three patients from the ISB-CC group were sent home with a disposable elastomeric pump that delivered a continuous infusion of $0.2 \%$ ropivacaine at $6 \mathrm{~mL} / \mathrm{hr}$ until completion of the allotted volume $(400 \mathrm{~mL})$.

Demographic data for each group of patients is provided in Table 1. Overall, there was no significant difference between the two groups in relation to age or BMI. The distribution of gender (male/female) as well as ASA physical status among the two groups was also very similar, with the exception of the ISB-LB group which had two ASA 1 patients, while there were none in the ISB-CC group (Table 1). In Table 4, the perioperative use of fentanyl, ketamine, and dexmedetomidine is shown for each patient population. No significant difference between the two groups was observed.

\section{Postoperative Pain Scores}

In Table 5, median pain scores during the 48-hour postoperative period are displayed for both ISB-CC and ISB-LB patients, with approximate reporting intervals of 6 hours, 12 hours, 24 hours, 36 hours, and 48 hours following ISB or PACU arrival time for those patients who received preoperative nerve blocks. For the ISB-CC group, the median pain score at 6 hours was 0 (interquartile range $0-4 / 10$ ), at 12 hours was 1 (interquartile range of $0-5 / 10$ ), at 24 hours was 2 (interquartile

Table 5 Postoperative Pain Scores - NPS with Interquartile Ranges

\begin{tabular}{|c|c|c|c|c|c|}
\hline & $\mathbf{6}$ Hours & $\mathbf{1 2}$ Hours & $\mathbf{2 4}$ Hours & $\mathbf{3 6}$ Hours & $\mathbf{4 8}$ Hours \\
\hline ISB-CC & & & & & \\
Median & $\mathbf{0}$ & $\mathbf{I}$ & $\mathbf{2}$ & $\mathbf{1}$ & $\mathbf{1 . 5}$ \\
$25^{\mathrm{TH}} \%$ & 0 & 0 & 0 & 0 & 0 \\
$75^{\mathrm{TH} \%}$ & 4 & 5 & 4 & 6.5 & 4.5 \\
$n$ & 19 & 19 & 15 & 12 & 9 \\
\hline ISB-LB & & & & & \\
Median & $\mathbf{0}$ & $\mathbf{2}$ & $\mathbf{3 . 5}$ & $\mathbf{0}$ & $\mathbf{0}$ \\
$25^{\mathrm{TH}} \%$ & 0 & 0 & 0 & 0 & 0 \\
$75^{\mathrm{TH}} \%$ & 3 & 7 & 7 & 2 & 2 \\
$n$ & 22 & 19 & 16 & 10 & 9 \\
\hline P-value & 0.94 & 0.70 & 0.40 & 0.32 & 0.45 \\
\hline
\end{tabular}

Notes: All statistical analysis performed with Wilcoxon rank-sum test. Italics: number of patients. Bold: median pain score.

Abbreviation: $\mathrm{n}$, number of patients. range $0-4 / 10$ ), at 36 hours was 1 (interquartile range $0-6.5 / 10$ ), and at 48 hours was 1.5 (interquartile range $0-4.5 / 10$ ). In comparison, for the ISB-LB group, the median numeric pain scores at 6 hours was 0 (interquartile range $0-3 / 10$ ), at 12 hours was 2 (interquartile range of $0-7 / 10$ ), at 24 hours was 3.5 (interquartile range $0-7 / 10$ ), at 36 hours was 0 (interquartile range $0-2 / 10$ ), and at 48 hours was 0 (interquartile range $0-2$ / 10). Upon analysis of these results, there appears to be no significant difference in median pain scores during the 48hour postoperative period when comparing the two patient groups (ISB-CC and ISB-LB).

\section{Postoperative Opioid Consumption}

Median opioid consumption (measured in MME) over the course of postoperative days (POD) 0-2 were calculated for both groups of patients. Opioid consumption reported in Table 6 and displayed in Figure 2 encompasses a 48-hour postoperative period. For the ISB-CC group, the median opioid consumption on POD 0 was 22.5 MME (interquartile range of 12-45 MME), on POD 1 was $30 \mathrm{MME}$ (interquartile range of 0-52.5 MME), and on POD 2 was $30 \mathrm{MME}$ (interquartile range of $0-45 \mathrm{MME}$ ). On the other hand, for the ISB-LB group, the median opioid consumption on POD 0 was 28.5 MME (interquartile range of 11-39.25 MME), on POD 1 was 37.5 MME (interquartile range of 15-47.5 MME), and on POD 2 was $25 \mathrm{MME}$ (interquartile range of 15-40 MME). Based on these results, there was no significant difference noted between the ISB-CC and ISB-LB patient groups in relation to median opioid consumption during the 48-hour postoperative period.

\section{Time Duration to Complete Regional Block Intervention}

The time required (measured in minutes) to complete each regional block intervention was recorded for both the ISB$\mathrm{CC}$ and ISB-LB groups as part of a secondary comparison. The median block time with interquartile ranges is displayed in Figure 3. For the ISB-CC group, the median block time was 9 minutes (interquartile range of 6-16 minutes) while for the ISB-LB group, the median block time was 3.5 minutes (interquartile range of 2-5 minutes). These results showed a significant difference $(\mathrm{p}<0.001)$ in time required to complete each regional block intervention (ISB-CC versus ISB-LB).

\section{Complications}

For the ISB-CC group, there were three documented complications related to catheter placement and maintenance (Table 7). In one patient with significant comorbidities 
Table 6 Postoperative Opioid Consumption - MME with Interquartile Ranges

\begin{tabular}{|l|c|c|c|}
\hline & POD 0 & POD I & POD 2 \\
\hline ISB-CC & & & \\
Median & $\mathbf{2 2 . 5}$ & $\mathbf{3 0}$ & $\mathbf{3 0}$ \\
$25^{\mathrm{TH}} \%$ & 12 & 0 & 0 \\
$75^{\mathrm{TH}} \%$ & 45 & 52.5 & 45 \\
$n$ & 19 & 19 & 11 \\
\hline ISB-LB & & & \\
Median & $\mathbf{2 8 . 5}$ & $\mathbf{3 7 . 5}$ & $\mathbf{2 5}$ \\
$25^{\mathrm{TH}} \%$ & 11 & 15 & 15 \\
$75^{\mathrm{TH}} \%$ & 39.25 & 47.5 & 40 \\
$n$ & 24 & 19 & 10 \\
\hline P-value & 0.96 & 0.46 & 0.86 \\
\hline
\end{tabular}

Notes: All statistical analysis performed with Wilcoxon rank-sum test. Italics: number of patients. Bold: median opioid consumption.

Abbreviation: $\mathrm{n}$, number of patients.

(ASA 3), there was persistent bleeding at the ISB catheter insertion site into the skin, and thus the decision was made to remove the catheter after injecting a bolus of $20 \mathrm{~mL}$ of $0.25 \%$ bupivacaine hydrochloride with $3 \mathrm{mg}$ of preservative-free dexamethasone. For this same patient, the PACU stay was complicated by respiratory distress requiring supplemental oxygen and point-of-care ultrasound examination revealed ipsilateral diaphragmatic hemiparesis from the ISB as well as small pre-existing bilateral pleural effusions. In a second patient, the indwelling catheter needed to be removed on POD 1 secondary to persistent neuropathic pain in the C5C6 dermatome distribution. This patient subsequently required a repeat single-shot ISB with $20 \mathrm{~mL}$ of $0.25 \%$ bupivacaine hydrochloride with $3 \mathrm{mg}$ of preservative-free dexamethasone. Finally, in a third patient, there was concern for possible catheter dislodgement based on inadequate clinical effect even after the catheter tip position was re-confirmed on ultrasound examination. As a result, the catheter was removed on POD 1 due to likely malfunctioning catheter equipment. For the ISB-LB group, there were no observed complications related to the effects of the single-shot ISB technique and no reported events of cardiac, neurological, or pulmonary compromise. For both patient groups, there were no reported return visits to the emergency room or readmissions for complications related to regional anesthetic intervention or complaints of uncontrolled pain.

\section{Discussion}

ISB-LB is a promising technique for providing extended shoulder analgesia, despite limited published data comparing its analgesic effectiveness to indwelling continuous interscalene catheters or local infiltration of liposomal

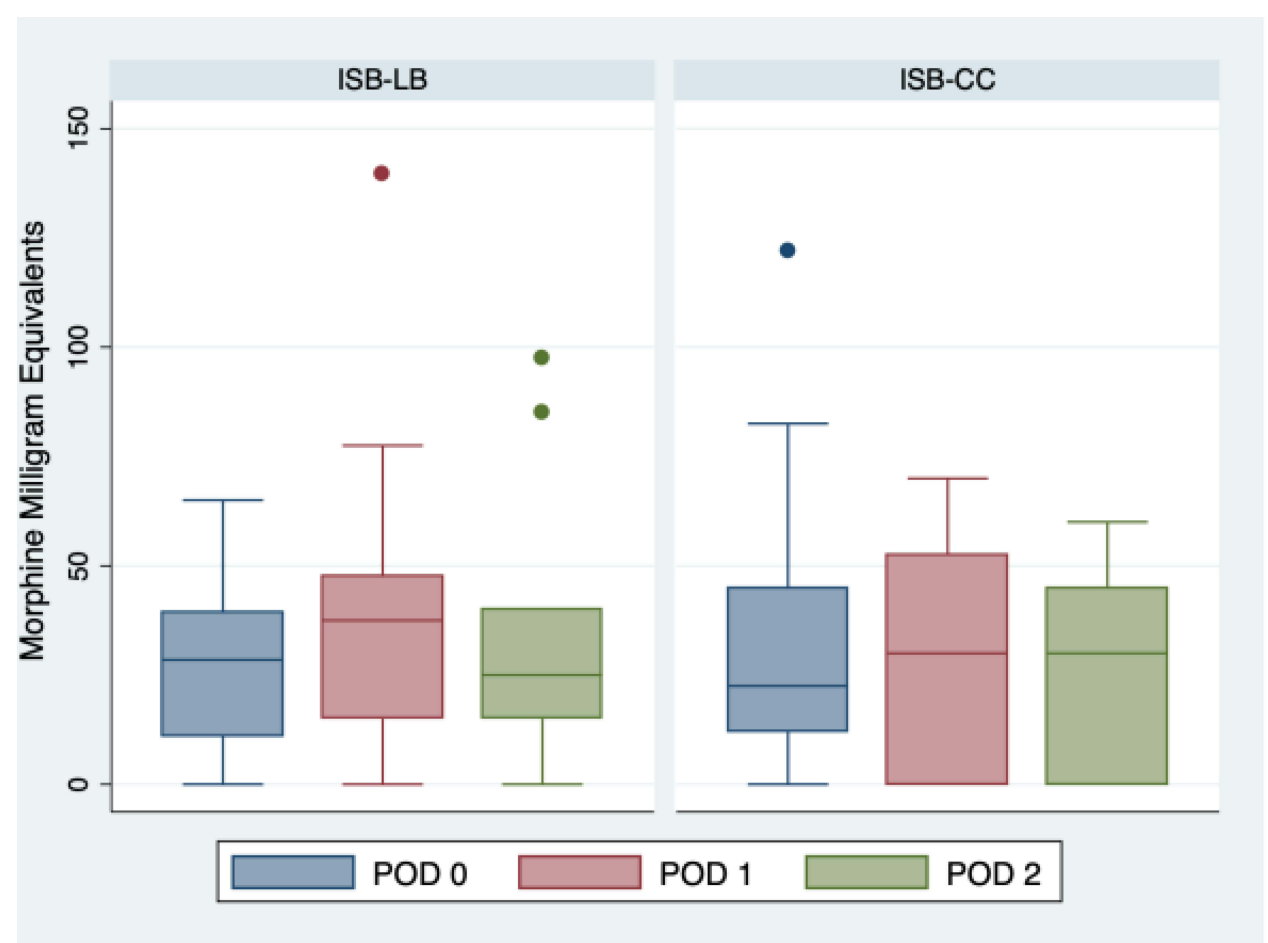

Figure 2 Box plot of postoperative opioid consumption in morphine milligram equivalents. Abbreviation: POD, postoperative day. 


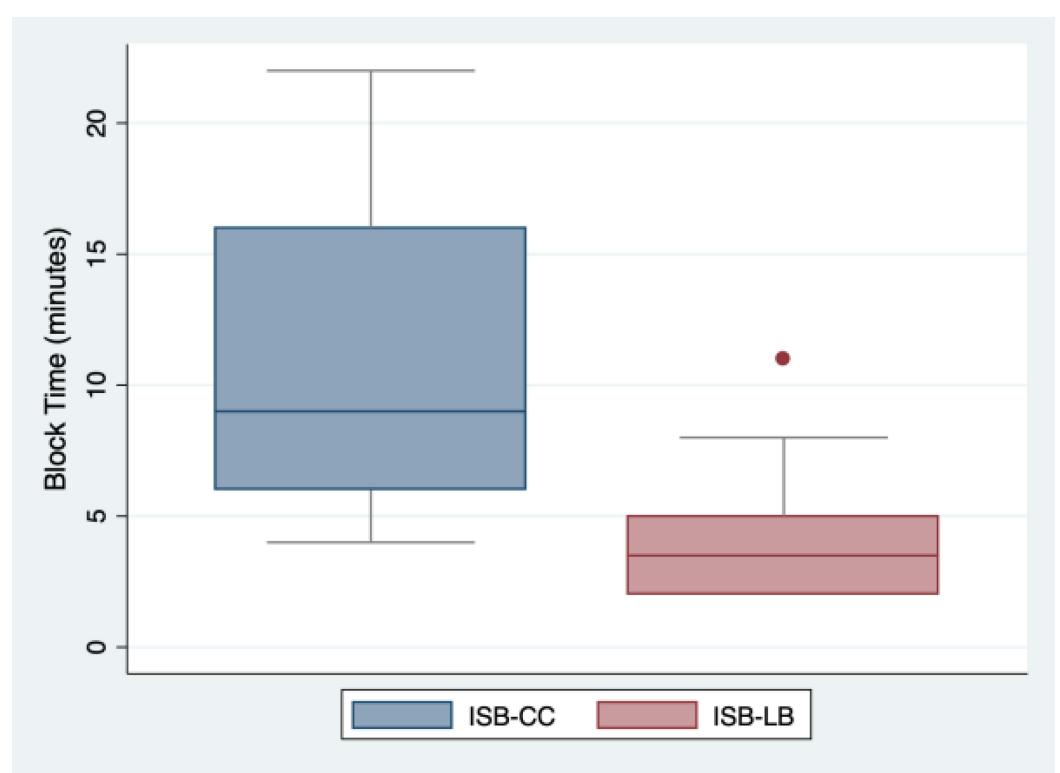

Figure 3 Box plot of time duration to complete regional block intervention.

bupivacaine during shoulder surgery. ${ }^{15,16}$ Effective multimodal management of postoperative pain following major shoulder surgery is essential to increase patient satisfaction, reduce hospital stay and costs, improve postoperative recovery and functional outcomes, as well as decrease risk for development of chronic pain.

The use of ISB-CC has been extensively studied and is a common technique for providing extended analgesia following shoulder surgery to minimize rebound pain and hospital length of stay, as well as to increase patient satisfaction. ${ }^{17-19}$ The use of single-shot ISB, even with additives such as dexamethasone, only provides up to approximately 22 hours of analgesia, thus placing the patient at risk of rebound pain and the need to rely on oral or intravenous opioids for further pain control. ${ }^{3,7,8}$ The benefits of using an indwelling continuous catheter system include the ability to titrate the rate of continuous infusion, administer boluses of local anesthetic, and stop or restart the infusion as needed to achieve the desired clinical effect during the postoperative recovery period.

However, the use of ISB continuous catheters has been associated with a significant number of potential complications. $^{20}$ There have been reports of significant increased relative risk (almost four times higher) of major complications with utilization of indwelling interscalene catheters, including respiratory distress secondary to prolonged phrenic nerve blockade, pneumonia, persistent neuritis, cardiac events, catheter incarceration, and catheter malfunctions such as leakage, dislodgement and clogging. ${ }^{21}$ In a study evaluating 1505 patients receiving continuous interscalene catheters following outpatient shoulder surgery, patients reported mild dyspnea (27\%),

Table 7 Complications After Interscalene Brachial Plexus Blockade

\begin{tabular}{|l|c|c|}
\hline Complication & ISB-CC Group, $\mathbf{n}$ (\% of Total) & ISB-LB Group, $\mathbf{n}$ (\% of Total) \\
\hline Dyspnea/Respiratory compromise & I (5.3)* & $0(0)$ \\
Superficial bleeding or skin reaction & I $(5.3)^{*}$ & $0(0)$ \\
Paresthesia/Neuralgia & $\mathrm{I}(5.3)$ & $0(0)$ \\
Catheter dislodgement/malfunction & $\mathrm{I}(5.3)$ & $0(0)$ \\
Horner syndrome (ptosis/miosis/anhidrosis) & $0(0)$ & $0(0)$ \\
Cardiovascular instability & $0(0)$ & $0(0)$ \\
Readmission for delayed complications & $0(0)$ & $0(0)$ \\
Readmission for pain & $0(0)$ & 0 \\
\hline
\end{tabular}

Note: *Both complications were experienced with the same patient. Abbreviation: $\mathrm{n}$, number of patients. 
numbness and/or tingling in the neck/arm/hand (14\%), catheter site erythema, pain, or discharge $(8.5 \%)$, and prolonged hand weakness after 1 week following catheter placement $(5 \%){ }^{22}$

Therefore, despite the proven effectiveness of ISB-CC to prolong analgesia following shoulder surgery, there is a concern for a variety of potential complications. Of note, in our study, there were 3 patients with complications observed in the ISB-CC group, which included a malfunctioning catheter, bleeding at the catheter insertion site, cervical neuropathy, and respiratory depression secondary to diaphragmatic hemiparesis in the setting of pre-existing small bilateral pleural effusions (Table 7). In addition, 3 patients from the ISB-CC group required a bolus through the catheter or a repeat interscalene rescue block upon removal of the catheter. These events and/or complications did not occur in the ISB-LB group of patients, despite a larger cohort. Thus, the risk factors associated with ISB-CC, in combination with the time required to place indwelling catheters and the need for skilled anesthesiologists has led to a search for alternative regional techniques that can minimize risk while also providing the benefits of extended analgesia following ISB.

Several studies have evaluated the utility of local periarticular infiltration of liposomal bupivacaine (LB) for shoulder surgery, but no clear consensus has been reached on the relative effectiveness and duration of analgesia provided with this technique, likely due to a heterogeneity of methods for intervention. ${ }^{15,21,23,24}$ In one randomized controlled trial, the use of LB as a local peri-articular injection during surgery was shown to offer minimal clinical benefit, if any, when used as adjuvant technique for pain control in addition to single-shot ISB. ${ }^{25}$ Thus, peri-articular injection of LB as an alternative to single-shot ISB or ISB-CC has shown inconsistent results and is not a reliable analgesic technique. ${ }^{23,26}$ However, the use of ISB-LB has been shown to be superior to placebo in a prospective, doubleblinded randomized controlled trial. ${ }^{27}$ In addition, a small prospective study of 52 patients demonstrated that combining LB with bupivacaine hydrochloride for ISB in the setting of major shoulder surgery resulted in better patient satisfaction and a modest improvement in pain scores over the course of the first postoperative week when compared to ISB with only bupivacaine hydrochloride. ${ }^{28}$ Pharmacokinetic studies demonstrate that plasma concentrations of bupivacaine peak after 12-36 hours following administration of LB, thus adding bupivacaine hydrochloride to the LB injectate provides analgesia during the time window of initial release of bupivacaine hydrochloride from the liposomes. ${ }^{29}$
In regard to the use of liposomal bupivacaine with ISB, Patel et al reported no observed complications with this technique as it relates to respiratory distress or need for supplemental oxygen, which is something that has been distinctly noted with the use of an ISB-CC. ${ }^{27}$ This may be due to the larger amounts of local anesthetic with catheters from the combined effect of single-shot injections, infusions, and boluses. In addition, a retrospective chart review of 1518 patients undergoing a variety of upper extremity surgical interventions noted no difference in complications when comparing ISB-LB to ISB with bupivacaine hydrochloride. ${ }^{30}$ However, in a recent retrospective review of 352 patients who received ISB-LB after undergoing shoulder surgery, postoperative complications related to prolonged clinical effects of the intervention were noted in $16.5 \%$ of patients, with $6 \%$ of patients requiring a return to the emergency department and half of these patients requiring readmission for supportive care. ${ }^{31}$ Most common symptoms included dyspnea and chest pain (12.5\%) and a number of patients reporting swelling, dermatitis, and hematomas at the site of ISB injection (1.7\%). It was noted that higher ASA physical status score was the strongest predictor of the occurrence of complications, and this encompassed patients with a history of cardiopulmonary disease and advanced age. That particular study demonstrates that ISB-LB is not free of complications or side effects. Patient selection is important and close monitoring is essential to evaluate for these clinical effects prior to discharge.

A recent retrospective review evaluated postoperative opioid consumption following total shoulder arthroplasty in patients who received preoperative ISB-LB as compared to an ISB-CC. ${ }^{32}$ The authors reported that the ISBLB patients had significantly lower postoperative opioid consumption during the first 24 hours and shorter total hospital length of stay when compared to the patients with ISB-CC, but no significant difference in mean pain scores after the initial 4 hours. Although the difference in mean opioid consumption in opioid-naive patients (33.2 MME vs 21.1 MME) achieved statistical significance in this study, clinically this translates to a difference of 12 MME which is approximately one or two oxycodone $5 \mathrm{mg}$ tablets. The mean hospital stay for these patients was 29.3 hours, thus not providing enough information about the extended analgesic benefit of these regional techniques in the immediate postoperative period. However, the ISB-LB group had a significantly lower 8-week postoperative 
opioid consumption (402 MME versus $582 \mathrm{MME}$ ) when compared to the ISB-CC group.

Our goal was to further examine the magnitude of extended analgesia provided by ISB-LB and ISB-CC through evaluating pain scores and opioid consumption during the 48 hours postoperatively in the setting of shoulder trauma requiring open shoulder surgery. The results indicate that ISB-LB provided comparable postoperative pain relief as compared to ISB-CC when measuring verbal pain scores and opioid consumption during the 48 -hour postoperative period. In addition, ISB-LB resulted in no major or minor complications, while three patients from the ISB-CC group experienced some level of setback related to catheter malfunction, bleeding at insertion site, neuralgia, and hemidiaphragmatic paresis (Table 7).

There are many advantages to single-shot ISBs over the use of ISB indwelling continuous catheters. First, it takes less time to administer a single-shot peripheral nerve block when compared to the more extensive time commitment required for placement, confirmation, and securing of catheters. In our study, we observed a significant difference in time required to complete the regional block intervention. Blocks administered for the ISB-LB group required a shorter overall time (median time of 3.5 minutes) as compared to the ISB-CC group (median time of 9 minutes). In addition, there is more technical skill and training required for ISB-CC placement. Management of catheters also requires a service that can attend to phone calls in an outpatient setting or an established acute pain team to manage and troubleshoot ISB-CC complications in an inpatient setting. One potential advantage of ISB-LB over ISB-CC in our trauma patients is the ability to administer the local anesthetic more distally along the superior trunk, which may provide extended analgesia with a potentially lower incidence of diaphragmatic paresis. ${ }^{33}$ Multi-orifice catheters do not provide the opportunity to direct the local anesthetic more distally in a precise fashion. On the other hand, a major disadvantage of ISB-LB is the finite duration of action with no ability to titrate further analgesia to clinical effect following the initial administration. There is theoretical concern for prolonged phrenic nerve palsy following ISB-LB, however, in our patient population, we did not observe any instances of respiratory compromise or readmissions due to complications following ISB-LB.

Although we can draw some conclusions from our retrospective study, there are certain limitations that are important to note. These include the retrospective nature of the study and the reviewer bias that accompanies it. Also, the lack of homogeneity between the two study groups in regards to surgery type as well as coexisting injuries is expected with retrospective reviews of polytrauma patients at a large level 1 trauma center. As a result, this can introduce additional variables to the perioperative analgesic requirements and extent of recovery for each individual patient. However, in our experience, the postoperative pain trajectories for surgical procedures evaluated in this study are similar enough for comparison in order to draw meaningful conclusions. For all shoulder operations included in this study, postoperative pain scores tend to be in the severe range and require intense analgesic management for an extended period of time. Of note, a similar pain trajectory has been demonstrated for arthroscopic shoulder surgery, with significant postoperative pain lasting for up to 72 hours. ${ }^{34}$

Another limitation of this study was the inability to evaluate long-term outcomes of ISB-CC or ISB-LB patients due to insufficient data after the 48-hour postoperative window and the lack of detailed follow-up documentation after discharge. On another note, since our patient population consisted of trauma patients, the presence of contralateral lung pathology served as a contraindication to ISB in several cases, necessitating phrenic-sparing analgesic strategies and thus limiting the inclusion of certain patients in our review.

Despite the limited sample size provided by the retrospective nature of this study and the set time during which patients were observed, the results provide insight into the clinical efficacy of both ISB regional techniques in patients undergoing shoulder surgery. Even amongst a small sample size, the bounds of the upper quartile opioid consumption only differed by approximately 5 MME between both groups on each postoperative day. This translates to only a subtle difference in opioid utilization in the clinical setting. Additionally, there was no consistent trend in pain scores that would suggest superiority of one technique over the other had there been a larger sample size. This retrospective study provides important initial data that highlights the comparable efficacy of both ISB regional techniques and allows for powering of a future prospective, randomized study.

\section{Conclusion}

In summary, this retrospective study provides valuable information about the efficacy of liposomal bupivacaine and continuous catheter systems for interscalene brachial plexus block in patients undergoing shoulder surgery. It is the first study to evaluate and compare these two regional 
anesthetic techniques in the trauma setting. However, it would still be essential to review a larger sample size of patients in order to establish further conclusions as well as confirm these findings with prospective studies. Ultimately, both ISB techniques resulted in comparable postoperative pain scores and opioid consumption within the 48-hour postoperative period, with a shorter time required to complete the regional intervention in the ISBLB group. Thus, ISB with liposomal bupivacaine may be a viable alternative to ISB indwelling continuous catheters to provide extended analgesia in patients undergoing major shoulder surgery.

\section{Disclosure}

The authors report no conflicts of interest in this work.

\section{References}

1. Cozowicz C, Poeran J, Memtsoudis SG. Epidemiology, trends, and disparities in regional anaesthesia for orthopaedic surgery. $\mathrm{Br}$ $J$ Anaesth. 2015;115(Suppl 2):ii57-67. doi:10.1093/bja/aev381

2. Egol KA, Forman J, Ong C, Rosenberg A, Karia R, Zuckerman JD. Regional anesthesia improves outcome in patients undergoing proximal humerus fracture repair. Bull Hosp Jt Dis. 2014;72(3):231-236.

3. Abdallah FW, Halpern SH, Aoyama K, Brull R. Will the real benefits of single-shot interscalene block please stand up? A systematic review and meta-analysis. Anesth Analg. 2015;120(5):1114-1129. doi:10.1213/ANE.0000000000000688

4. Bishop JY, Sprague M, Gelber J, et al. Interscalene regional anesthesia for shoulder surgery. J Bone Joint Surg Am. 2005;87(5):974-979. doi:10.2106/JBJS.D.02003

5. Chalmers PN, Salazar D, Fingerman ME, Keener JD, Chamberlain A. Continuous interscalene brachial plexus blockade is associated with reduced length of stay after shoulder arthroplasty. Orthop Traumatol Surg Res. 2017;103(6):847-852. doi:10.1016/j.otsr.2017.06.007

6. Goebel S, Stehle J, Schwemmer U, Reppenhagen S, Rath B, Gohlke F. Interscalene brachial plexus block for open-shoulder surgery: a randomized, double-blind, placebo-controlled trial between single-shot anesthesia and patient-controlled catheter system. Arch Orthop Trauma Surg. 2010;130(4):533-540. doi:10.1007/s00402009-0985-7

7. Cummings KC 3rd, Napierkowski DE, Parra-Sanchez I, et al. Effect of dexamethasone on the duration of interscalene nerve blocks with ropivacaine or bupivacaine. $\mathrm{Br} J$ Anaesth. 2011;107(3):446-453. doi:10.1093/bja/aer159

8. Kirkham KR, Jacot-Guillarmod A, Albrecht E. Optimal dose of perineural dexamethasone to prolong analgesia after brachial plexus blockade: a systematic review and meta-analysis. Anesth Analg. 2018;126(1):270-279. doi:10.1213/ANE.0000000000002488

9. Lavand'homme P. Rebound pain after regional anesthesia in the ambulatory patient. Curr Opin Anaesthesiol. 2018;31(6):679-684. doi:10.1097/ACO.0000000000000651

10. Soffer RJ, Rosenblatt MA. Teaching ultrasound-guided interscalene blocks: description of a simple and effective technique. J Clin Anesth. 2007;19(3):241-242. doi:10.1016/j.jclinane.2007.02.001

11. Casati A, Vinciguerra F, Scarioni M, et al. Lidocaine versus ropivacaine for continuous interscalene brachial plexus block after open shoulder surgery. Acta Anaesthesiol Scand. 2003;47(3):355-360. doi:10.1034/j.1399-6576.2003.00065.x
12. Nielsen S, Degenhardt L, Hoban B, Gisev N. A synthesis of oral morphine equivalents (OME) for opioid utilisation studies. Pharmacoepidemiol Drug Saf. 2016;25(6):733-737. doi:10.1002/ pds. 3945

13. Hu Z, Qin J. Generalizability of causal inference in observational studies under retrospective convenience sampling. Stat Med. 2018;37 (19):2874-2883. doi:10.1002/sim.7808

14. Guttman OT, Soffer RJ, Rosenblatt MA. The ultrasound-guided supraclavicular-interscalene (UGSCIS) block: a case report. Pain Pract. 2008;8(1):62-64. doi:10.1111/j.1533-2500.2007.00156.x

15. Kolade O, Patel K, Ihejirika R, et al. Efficacy of liposomal bupivacaine in shoulder surgery: a systematic review and meta-analysis. $J$ Shoulder Elbow Surg. 2019;28(9):1824-1834. doi:10.1016/j. jse.2019.04.054

16. Orebaugh SL, Dewasurendra A. Has the future arrived? Liposomal bupivacaine versus perineural catheters and additives for interscalene brachial plexus block. Curr Opin Anaesthesiol. 2020;33(5):704-709. doi:10.1097/ACO.0000000000000913

17. Mariano ER, Afra R, Loland VJ, et al. Continuous interscalene brachial plexus block via an ultrasound-guided posterior approach: a randomized, triple-masked, placebo-controlled study. Anesth Analg. 2009;108(5):1688-1694. doi:10.1213/ane.0b013e318199dc86

18. Salviz EA, Xu D, Frulla A, et al. Continuous interscalene block in patients having outpatient rotator cuff repair surgery: a prospective randomized trial. Anesth Analg. 2013;117(6):1485-1492. doi:10.1213/01.ane.0000436607.40643.0a

19. Vorobeichik L, Brull R, Bowry R, Laffey JG, Abdallah FW. Should continuous rather than single-injection interscalene block be routinely offered for major shoulder surgery? A meta-analysis of the analgesic and side-effects profiles. $B r J$ Anaesth. 2018;120(4):679-692. doi:10.1016/j.bja.2017.11.104

20. Lenters TR, Davies J, Matsen FA 3rd. The types and severity of complications associated with interscalene brachial plexus block anesthesia: local and national evidence. J Shoulder Elbow Surg. 2007;16(4):379-387. doi:10.1016/j.jse.2006.10.007

21. Weller WJ, Azzam MG, Smith RA, Azar FM, Throckmorton TW. Liposomal bupivacaine mixture has similar pain relief and significantly fewer complications at less cost compared to indwelling interscalene catheter in total shoulder arthroplasty. J Arthroplasty. 2017;32 (11):3557-3562. doi:10.1016/j.arth.2017.03.017

22. Fredrickson MJ, Leightley P, Wong A, Chaddock M, Abeysekera A, Frampton C. An analysis of 1505 consecutive patients receiving continuous interscalene analgesia at home: a multicentre prospective safety study. Anaesthesia. 2016;71(4):373-379. doi:10.1111/ anae. 13385

23. Abildgaard JT, Lonergan KT, Tolan SJ, et al. Liposomal bupivacaine versus indwelling interscalene nerve block for postoperative pain control in shoulder arthroplasty: a prospective randomized controlled trial. J Shoulder Elbow Surg. 2017;26(7):1175-1181. doi:10.1016/j. jse.2017.03.012

24. Sabesan VJ, Shahriar R, Petersen-Fitts GR, et al. A prospective randomized controlled trial to identify the optimal postoperative pain management in shoulder arthroplasty: liposomal bupivacaine versus continuous interscalene catheter. J Shoulder Elbow Surg. 2017;26(10):1810-1817. doi:10.1016/j.jse.2017.06.044

25. Namdari S, Nicholson T, Abboud J, Lazarus M, Steinberg D, Williams G. Interscalene block with and without intraoperative local infiltration with liposomal bupivacaine in shoulder arthroplasty: a randomized controlled trial. J Bone Joint Surg Am. 2018;100 (16):1373-1378. doi:10.2106/JBJS.17.01416

26. Namdari S, Nicholson T, Abboud J, Lazarus M, Steinberg D, Williams G. Randomized controlled trial of interscalene block compared with injectable liposomal bupivacaine in shoulder arthroplasty. $J$ Bone Joint Surg Am. 2017;99(7):550-556. doi:10.2106/ JBJS.16.00296 
27. Patel MA, Gadsden JC, Nedeljkovic SS, et al. Brachial plexus block with liposomal bupivacaine for shoulder surgery improves analgesia and reduces opioid consumption: results from a multicenter, randomized, double-blind, controlled trial. Pain Med. 2020;21(2):387-400. doi:10.1093/pm/pnz103

28. Vandepitte C, Kuroda M, Witvrouw R, et al. Addition of liposome bupivacaine to bupivacaine $\mathrm{HCl}$ versus bupivacaine $\mathrm{HCl}$ alone for interscalene brachial plexus block in patients having major shoulder surgery. Reg Anesth Pain Med. 2017;42(3):334-341. doi:10.1097/ AAP.0000000000000560

29. Hu D, Onel E, Singla N, Kramer WG, Hadzic A. Pharmacokinetic profile of liposome bupivacaine injection following a single administration at the surgical site. Clin Drug Investig. 2013;33(2):109-115. doi:10.1007/s40261-012-0043-z

30. Hutchins JL, Habeck J, Novaczyk Z, et al. Patient complications after interscalene block: a retrospective comparison of liposomal bupivacaine to nonliposomal bupivacaine. Anesthesiol Res Pract. 2020;2020:6704303. doi:10.1155/2020/6704303
31. Malige A, Yeazell S, Ng-Pellegrino A, Carolan G. Risk factors for complications and return to the emergency department after interscalene block using liposomal bupivacaine for shoulder surgery. J Shoulder Elbow Surg. 2020;29(11):2332-2338. doi:10.1016/j. jse.2020.03.012

32. Weir TB, Simpson N, Aneizi A, et al. Single-shot liposomal bupivacaine interscalene block versus continuous interscalene catheter in total shoulder arthroplasty: opioid administration, pain scores, and complications. J Orthop. 2020;22:261-267. doi:10.1016/j.jor.2020.05.006

33. Kim DH, Lin Y, Beathe JC, et al. Superior trunk block: a phrenic-sparing alternative to the interscalene block: a randomized controlled trial. Anesthesiology. 2019;131(3):521-533. doi:10.1097/ ALN.0000000000002841

34. Mariano ER, El-Boghdadly K, Ilfeld BM. Using postoperative pain trajectories to define the role of regional analgesia in personalised pain medicine. Anaesthesia. 2021;76(2):165-169. doi:10.1111/ anae. 15067

\section{Publish your work in this journal}

Local and Regional Anesthesia is an international, peer-reviewed, open access journal publishing on the development, pharmacology, delivery and targeting and clinical use of local and regional anesthetics and analgesics. The journal welcomes submitted papers covering original research, basic science, clinical studies, reviews \& evaluations, guidelines, expert opinion and commentary, case reports and extended reports. The manuscript management system is completely online and includes a very quick and fair peer-review system, which is all easy to use. Visit http://www.dovepress.com/testimonials. php to read real quotes from published authors. 